NASA/CR-1998-207935
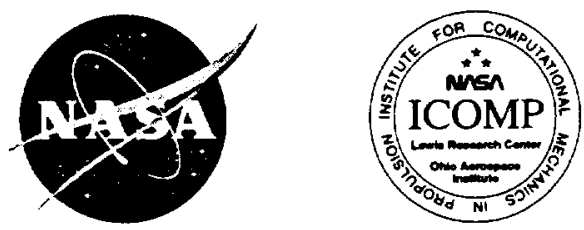

\title{
A Formulation of Asymptotic and Exact Boundary Conditions Using Local Operators
}

T. Hagstrom

The University of New Mexico, Albuquerque, New Mexico

S.I. Hariharan

The University of Akron, Akron, Ohio

Prepared under Cooperative Agreement NAG3-2014

National Aeronautics and

Space Administration

Lewis Research Center 
Available from

NASA Center for Aerospace Information 7121 Standard Drive Hanover, MD 21076

Price Code: A03
National Technical Information Service 5287 Port Royal Road Springfield, VA 22100 Price Code: $\mathrm{A} 03$ 


\title{
A Formulation of Asymptotic and Exact Boundary Conditions Using Local Operators
}

\author{
Thomas Hagstrom ${ }^{1}$ \\ Department of Mathematics and Statistics \\ The University of New Mexico \\ Albuquerque, NM, USA \\ S. I. Hariharan ${ }^{2}$ \\ Department of Mathematical Sciences \\ The University of Akron \\ Akron, $\mathrm{OH}, \mathrm{USA}$
}

\begin{abstract}
In this paper we describe a systematic approach for constructing asymptotic boundary conditions for isotropic wave-like equations using local operators. The conditions take a recursive form with increasing order of accuracy. In three dimensions the recursion terminates and the resulting conditions are exact for solutions which are described by finite combinations of angular spherical harmonics. First, we develop the expansion for the two-dimensional wave equation and construct a sequence of easily implementable boundary conditions. We show that in three dimensions the analogous conditions are again easily implementable in addition to being exact. Also, we provide extensions of these ideas to hyperbolic systems. Namely, Maxwell's equations for TM waves are used to demonstrate the construction. Finally, we provide numerical examples to demonstrate the effectiveness of these conditions for a model problem governed by the wave equation.
\end{abstract}

\footnotetext{
${ }^{1}$ Supported in part by NSF Grant DMS-9600146 and by ICOMP, OAI, NASA Lewis Research Center, Cleveland, $\mathrm{OH}$. Work partially carried out while the author was visiting the Courant Institute of Mathematical Sciences, New York, NY.

${ }^{2}$ Supported in part by NASA Grant NCC3-494.
} 


\section{Introduction}

The topic of boundary conditions for wave-like equations has been an active area for the past two decades, with much work focusing on the development of sequences of conditions of increasing accuracy based on local operators. Growing interest in demanding problems in computational electromagnetics and aeroacoustics has increased the need for more accurate and efficient techniques. In this paper we discuss and derive a sequence of boundary conditions for isotropic problems. Preliminary results of this work are found in [8]. Generalizations to anisotropic problems, such as the linearized compressible Euler system, will be discussed elsewhere. The present development originates with the well-known reference [2]. It differs from that and from others in that conditions of arbitrary order are expressed recursively using auxiliary functions. This allows them to be implemented with great ease. It also allows the adaptive determination of the order of the boundary condition, which we will demonstrate elsewhere [7]. Moreover, in three space dimensions, the recursion truncates for finite spherical harmonic expansions, leading to an exact condition. This condition is similar to that of Grote and Keller [3, 4], but is somewhat easier and cheaper to use as it avoids spherical harmonic transformations.

This paper is divided into four parts. In section 2 we describe the procedure for the two-dimensional wave equation. The key idea is to construct a sequence of operators that approximately annihilate the residual of the preceding element in the sequence, viewed as a function on the artificial boundary. The sequence begins with the first order operator proposed in [2]. The recursive operators are constructed in such a way that they yield a stable class of conditions. The stability issue however is not discussed in this paper and will be reported elsewhere. Also, we indicate how these conditions can be approximated numerically. In section 3 we discuss analogous constructions for the three-dimensional wave equation. Generalizations to a hyperbolic system, that is Maxwell's equations in the TM case, are presented in section 4. Finally, in section 5 , we give some numerical results.

\section{The Two-Dimensional Wave Equation}

A formal series representation for an outgoing solution of the two-dimensional wave equation

$$
\frac{1}{c^{2}} \frac{\partial^{2} u}{\partial t^{2}}=\frac{\partial^{2} u}{\partial r^{2}}+\frac{1}{r} \frac{\partial u}{\partial r}+\frac{1}{r^{2}} \frac{\partial^{2} u}{\partial \theta^{2}}
$$

takes the form:

$$
\begin{gathered}
u=\sum_{n=0}^{\infty} a_{n}(\theta) f^{n}(r, t), \\
f^{n}=\sum_{k=0}^{\infty} r^{-k-1 / 2} f_{k}^{n}(c t-r),
\end{gathered}
$$


where,

$$
\left(f_{k+1}^{n}\right)^{\prime}=-\frac{\left(k+\frac{1}{2}\right)^{2}-n^{2}}{2(k+1)} f_{k}^{n}, \quad a_{n}^{\prime \prime}=-n^{2} a_{n}
$$

By setting

$$
\frac{1}{c} \frac{\partial u}{\partial t}+\frac{\partial u}{\partial r}+\frac{1}{2 r} u=w_{1}
$$

it is easily observed that

$$
\begin{gathered}
w_{1}=\sum_{n=0}^{\infty} a_{n}(\theta) f^{n, 1}(r, t), \\
f^{n, 1}=\sum_{k=1}^{\infty} r^{-k-3 / 2} \gamma_{k, 1} f_{k}^{n}(c t-r), \\
\gamma_{k, 1}=-k .
\end{gathered}
$$

By direct computation we note that $w_{1}=O\left(r^{-2}\right) u=O\left(r^{-5 / 2}\right)$. We now seek relations approximately satisfied by $w_{1}$, which in addition do not involve radial derivatives. In [8] we proposed a sequence of time derivatives of $w_{1}$ to derive higher order conditions. While they are accurate, we found them to be only marginally stable. Here we propose adding a damping term to the time derivative of $w_{1}$, as first suggested by Barry, Bielak and MacCamy [1]. Precisely, we apply the operator $\frac{1}{c} \frac{\partial}{\partial t}+\frac{\delta_{1}}{r}$ to $w_{1}$. This leads to the following calculations:

$$
\begin{aligned}
\frac{1}{c} \frac{\partial w_{1}}{\partial t}+\frac{\delta_{1}}{r} w_{1} & =-\sum_{n=0}^{\infty} a_{n} \sum_{k=0}^{\infty} r^{-k-5 / 2}\left((k+1)\left(f_{k+1}^{n}\right)^{\prime}+\delta_{1} k f_{k}^{n}\right) \\
& =\sum_{n=0}^{\infty} a_{n} \sum_{k=0}^{\infty} r^{-k-5 / 2}\left(\frac{1}{2}\left(\left(k+\frac{1}{2}\right)^{2}-n^{2}\right)-\delta_{1} k\right) f_{k}^{n} \\
& =\frac{1}{8 r^{2}} u+\frac{1}{2 r^{2}} \frac{\partial^{2} u}{\partial \theta^{2}}+\sum_{n=0}^{\infty} a_{n} \sum_{k=1}^{\infty} r^{-k-5 / 2}\left(\frac{1}{2}\left(k+\frac{1}{2}\right)^{2}-\delta_{1} k-\frac{1}{8}\right) f_{k}^{n}
\end{aligned}
$$

The remainder term is further reduced if $\delta_{1}$ is chosen to eliminate the first term:

$$
\begin{gathered}
\delta_{1}=1, \quad w_{2}=\sum_{n=0}^{\infty} a_{n} f^{n, 2} \\
f^{n, 2}=\sum_{k=2}^{\infty} r^{-k-5 / 2} \gamma_{k, 2} f_{k}^{n}(c t-r), \quad \gamma_{k, 2}=\frac{1}{2} k(k-1) .
\end{gathered}
$$

Clearly, we formally have $w_{2}=O\left(r^{-4}\right) u=O\left(r^{-9 / 2}\right)$. 
We now state and prove the general formula. Let

$$
\begin{gathered}
w_{j}=\sum_{n=0}^{\infty} a_{n}(\theta) f^{n, j}(r, t), \\
f^{n, j}=\sum_{k=j}^{\infty} r^{-k-j-1 / 2} \gamma_{k, j} f_{k}^{n}(c t-r),
\end{gathered}
$$

where

$$
\gamma_{k, j}=(-1)^{j} 2^{1-j} k(k-1) \cdots(k-(j-1)) .
$$

Then, for $j \geq 2$ :

$$
\frac{1}{c} \frac{\partial w_{j}}{\partial t}+\frac{j}{r} w_{j}=\frac{\left(j-\frac{1}{2}\right)^{2}}{4 r^{2}} w_{j-1}+\frac{1}{4 r^{2}} \frac{\partial^{2} w_{j-1}}{\partial \theta^{2}}+w_{j+1}, \quad j=2, \ldots
$$

To prove this we simply expand every term. On the left-hand side we have:

$$
-\sum_{k=j-1}^{\infty} r^{-k-j-3 / 2} \gamma_{k+1, j} \frac{\left(k+\frac{1}{2}\right)^{2}-n^{2}}{2(k+1)} f_{k}^{n}+\sum_{k=j}^{\infty} r^{-k-j-3 / 2} j \gamma_{k, j} f_{k}^{n} .
$$

On the right-hand side we have:

$$
\sum_{k=j-1}^{\infty} r^{-k-j-3 / 2} \frac{\left(j-\frac{1}{2}\right)^{2}-n^{2}}{4} \gamma_{k, j-1}+\sum_{k=j+1}^{\infty} r^{-k-j-3 / 2} \gamma_{k, j+1} f_{k}^{n}
$$

Separately we check $k=j-1, k=j$ and $k \geq j+1$.

$$
\begin{aligned}
-\gamma_{j, j} \frac{\left(j-\frac{1}{2}\right)^{2}-n^{2}}{2 j} & =(-1)^{j-1} 2^{1-j} j ! \frac{\left(j-\frac{1}{2}\right)^{2}-n^{2}}{2 j} \\
& =(-1)^{j-1} 2^{2-j}(j-1) ! \frac{\left(j-\frac{1}{2}\right)^{2}-n^{2}}{4} \\
& =\gamma_{j-1, j-1} \frac{\left(j-\frac{1}{2}\right)^{2}-n^{2}}{4} \\
-\gamma_{j+1, j} \frac{\left(j+\frac{1}{2}\right)^{2}-n^{2}}{2 j+2}+j \gamma_{j, j} & =(-1)^{j-1} 2^{1-j}(j+1) ! \frac{\left(j+\frac{1}{2}\right)^{2}-n^{2}}{2 j+2}-(-1)^{j-1} 2^{1-j} j ! j \\
& =(-1)^{j-1} 2^{2-j} j ! \frac{\left(j-\frac{1}{2}\right)^{2}-n^{2}}{4} \\
& =\gamma_{j, j-1} \frac{\left(j-\frac{1}{2}\right)^{2}-n^{2}}{4} .
\end{aligned}
$$




$$
\begin{aligned}
-\gamma_{k+1, j} \frac{\left(k+\frac{1}{2}\right)^{2}-n^{2}}{2(k+1)}+j \gamma_{k, j}= & (-1)^{j-1} 2^{1-j}(k+1) \cdots(k+2-j) \frac{\left(k+\frac{1}{2}\right)^{2}-n^{2}}{2(k+1)} \\
& -j(-1)^{j-1} 2^{1-j} k \cdots(k+1-j) \\
= & (-1)^{j-1} 2^{2-j} k \cdots(k+2-j) \frac{\left(j-\frac{1}{2}\right)^{2}-n^{2}+(k+1-j)(k-j)}{4} \\
= & \gamma_{k, j-1} \frac{\left(j-\frac{1}{2}\right)^{2}-n^{2}}{4}+\gamma_{k, j+1} .
\end{aligned}
$$

This recursion leads to the sequence of conditions:

$$
\begin{gathered}
\frac{1}{c} \frac{\partial u}{\partial t}+\frac{\partial u}{\partial r}+\frac{1}{2 r} u=w_{1}, \\
\frac{1}{c} \frac{\partial w_{j}}{\partial t}+\frac{j}{r} w_{j}=\frac{\left(j-\frac{1}{2}\right)^{2}}{4 r^{2}} w_{j-1}+\frac{1}{4 r^{2}} \frac{\partial^{2} w_{j-1}}{\partial \theta^{2}}+w_{j+1}, \quad j=1, \ldots
\end{gathered}
$$

where we have set

$$
w_{0}=2 u
$$

Formally,

$$
w_{j}=O\left(r^{-2 j}\right) u=O\left(r^{-(2 j+1 / 2)}\right)
$$

Using $p$ auxiliary functions involves setting $w_{p+1}=0$ which leads to an expected accuracy of $O\left(r^{-2 p-2}\right)$.

\section{The Three-Dimensional Wave Equation}

The wave equation in three dimensions is given by:

$$
\frac{1}{c^{2}} \frac{\partial^{2} u}{\partial t^{2}}=\frac{\partial^{2} u}{\partial r^{2}}+\frac{2}{r} \frac{\partial u}{\partial r}+\frac{1}{r^{2}} \nabla_{s}^{2} u
$$

where the spherical Laplacian is given by:

$$
\nabla_{s}^{2} u=\frac{1}{\sin \theta} \frac{\partial}{\partial \theta}\left(\sin \theta \frac{\partial u}{\partial \theta}\right)+\frac{1}{\sin ^{2} \theta} \frac{\partial^{2} u}{\partial \phi^{2}} .
$$

We seek a formal solution of the form:

$$
\begin{gathered}
u=\sum_{n=0}^{\infty} \sum_{m=0}^{n} a_{n, m}(\theta, \phi) f^{n, m}(r, t), \\
a_{n, m}=\left(A_{n, m} \cos m \phi+B_{n, m} \sin m \phi\right) P_{n}^{m}(\cos \theta),
\end{gathered}
$$




$$
f^{n, m}(r, t)=\sum_{k=0}^{\infty} r^{-k-1} f_{k}^{n, m}(c t-r) .
$$

Assuming each term in the first summation to be a solution, and recalling the fact that

$$
\nabla_{s}^{2} a_{n, m}=-n(n+1) a_{n, m},
$$

we derive the following recursion relation:

$$
\left(f_{k+1}^{n, m}\right)^{\prime}=-\frac{(k(k+1)-n(n+1))}{2(k+1)} f_{k}^{n, m}
$$

where $f_{0}^{n, m}$ is arbitrary. Note that we may choose $f_{k}^{n, m}=0$ for $k>n$ so that:

$$
f^{n, m}(r, t)=\sum_{k=0}^{n} r^{-k-1} f_{k}^{n, m}(c t-r) .
$$

Moreover, given $f^{n, m}(R, t)$, the right-hand side of (3.0.28) can be rewritten as an $n$th order differential operator on $f_{n}^{n, m}$,

$$
\begin{aligned}
& f^{n, m}(R, t)=\sum_{k=0}^{n} R^{-k-1} \eta_{k} \frac{1}{c^{n-k}} \frac{d^{n-k} f_{n}^{n, m}}{d t^{n-k}}(c t-R), \\
& \eta_{n}=1, \quad \eta_{k}=\prod_{j=k+1}^{n} \frac{2 j}{n(n+1)-j(j-1)}, \quad k<n .
\end{aligned}
$$

Hence, given $f^{n, m}(R, t)$, the expansion (3.0.28) certainly exists. We emphasize that we do not need to compute the functions $f_{k}^{n, m}$ in order to implement the boundary conditions. (See (3.0.47)-(3.0.48).)

We now repeat the derivation of the preceding section. The main difference will be the termination of the sequence for each finite $n$. Set,

$$
\begin{gathered}
\frac{1}{c} \frac{\partial u}{\partial t}+\frac{\partial u}{\partial r}+\frac{1}{r} u=w_{1} \\
w_{1}=\sum_{n=1}^{\infty} \sum_{m=0}^{n} a_{n, m}(\theta, \phi) f^{n, m, 1}(r, t), \\
f^{n, m, 1}=\sum_{k=1}^{n} r^{-k-2} \gamma_{k, 1} f_{k}^{n, m}(c t-r) .
\end{gathered}
$$

By direct computation we note that $w_{1}=O\left(r^{-2}\right) u=O\left(r^{-3}\right)$ and

$$
\gamma_{k, 1}=-k \text {. }
$$


Now consider:

$$
\begin{aligned}
\frac{1}{c} \frac{\partial w_{1}}{\partial t}+\frac{\delta_{1}}{r} w_{1} & =-\sum_{n=1}^{\infty} \sum_{m=0}^{n} a_{n, m} \sum_{k=0}^{n} r^{-k-3}\left((k+1)\left(f_{k+1}^{n, m}\right)^{\prime}+\delta_{1} k f_{k}^{n, m}\right) \\
& =\sum_{n=1}^{\infty} \sum_{m=0}^{n} a_{n, m} \sum_{k=0}^{n} r^{-k-3}\left(\frac{1}{2}(k(k+1)-n(n+1))-\delta_{1} k\right) f_{k}^{n, m}(3.0 \\
& =\frac{1}{2 r^{2}} \nabla_{s}^{2} u+\sum_{n=1}^{\infty} \sum_{m=0}^{n} a_{n, m} \sum_{k=1}^{n} r^{-k-3} \frac{1}{2} k\left(k+1-2 \delta_{1}\right) f_{k}^{n, m}
\end{aligned}
$$

The remainder term is further reduced if $\delta_{1}$ is chosen to eliminate the first term:

$$
\begin{gathered}
\delta_{1}=1, \quad w_{2}=\sum_{n=2}^{\infty} \sum_{m=0}^{n} a_{n, m} f^{n, m, 2}, \\
f^{n, m, 2}=\sum_{k=2}^{n} r^{-k-3} \gamma_{k, 2} f_{k}^{n, m}(c t-r), \quad \gamma_{k, 2}=\frac{1}{2} k(k-1) .
\end{gathered}
$$

Clearly, $w_{2}=O\left(r^{-4}\right) u=O\left(r^{-5}\right)$. Moreover, if we were in the special case of a solution in the span of the spherical harmonics of index less than 2 , then $w_{2}=0$ and the condition is exact.

We now consider the general case. Suppose for $1 \leq j \leq p, p \geq 2$,

$$
\begin{gathered}
w_{j}=\sum_{n=j}^{\infty} \sum_{m=0}^{n} a_{n, m}(\theta, \phi) f^{n, m, j}(r, t), \\
f^{n, m, j}=\sum_{k=j}^{n} r^{-k-j-1} \gamma_{k, j} f_{k}^{n, m}(c t-r),
\end{gathered}
$$

where again $\gamma_{k, j}$ is as given in (2.0.13). Then:

$$
\frac{1}{c} \frac{\partial w_{p}}{\partial t}+\frac{p}{r} w_{p}=\sum_{n=p}^{\infty} \sum_{m=0}^{n} a_{n, m}\left(\frac{1}{c} \frac{\partial f^{n, m, p}}{\partial t}+\frac{p}{r} f^{n, m, p}\right),
$$

and

$$
\begin{aligned}
\frac{1}{c} \frac{\partial f^{n, m, p}}{\partial t}+\frac{p}{r} f^{n, m, p} & =\sum_{k=p}^{n} r^{-k-p-1} \gamma_{k, p}\left(\left(f_{k}^{n, m}\right)^{\prime}+\frac{p}{r} f_{k}^{n, m}\right) \\
& =\sum_{k=p-1}^{n} r^{-k-p-2}\left(-\frac{\gamma_{k+1, p}}{2(k+1)}(k(k+1)-n(n+1))+p \gamma_{k, p}\right) f_{k}^{n, m}
\end{aligned}
$$


where we have used the fact that $\gamma_{p-1, p}=0$. Noting that:

$$
-\frac{\gamma_{k+1, p}}{2(k+1)}=\frac{1}{4} \gamma_{k, p-1}
$$

and

$$
\gamma_{k, p}=-\frac{(k-(p-1))}{2} \gamma_{k, p-1}
$$

we find

$$
\begin{aligned}
\frac{1}{c} \frac{\partial w_{p}}{\partial t}+\frac{p}{r} w_{p}= & \frac{1}{4 r^{2}}\left(\nabla_{s}^{2}+p(p-1)\right) w_{p-1} \\
& +\sum_{n=p-1}^{\infty} \sum_{m=0}^{n} a_{n, m} \sum_{k=p-1}^{n} r^{-k-p-2} \frac{\gamma_{k, p-1}}{4}(k-p)(k-(p-1)) f_{k}^{n, m}
\end{aligned}
$$

As the $k=p$ and $k=p-1$ terms in the final summation vanish, the $n=p-1$ and $n=p$ terms are eliminated. Moreover,

$$
\frac{\gamma_{k, p-1}}{4}(k-p)(k-(p-1))=\gamma_{k, p+1},
$$

so the remainder is given by:

$$
w_{p+1}=\sum_{n=p+1}^{\infty} \sum_{m=0}^{n} a_{n, m} \sum_{k=p+1}^{n} r^{-k-p-2} \gamma_{k, p+1} f_{k}^{n, m} .
$$

By induction we obtain:

$$
\begin{gathered}
\frac{1}{c} \frac{\partial u}{\partial t}+\frac{\partial u}{\partial r}+\frac{1}{r} u=w_{1} \\
\frac{1}{c} \frac{\partial w_{j}}{\partial t}+\frac{j}{r} w_{j}=\frac{1}{4 r^{2}}\left(\nabla_{s}^{2}+j(j-1)\right) w_{j-1}+w_{j+1}, \quad j=2, \ldots
\end{gathered}
$$

where $w_{0}=2 u, w_{j}$ satisfies (3.0.38)-(3.0.39) and, hence,

$$
w_{j}=O\left(r^{-2 j}\right) u=O\left(r^{-2 j-1}\right) .
$$

Using $p$ auxiliary functions involves setting $w_{p+1}=0$ which leads to an expected accuracy of $O\left(r^{-2 p-2}\right)$. However, in the special case:

$$
f^{n, m}=0, \quad n>p
$$

that is when the solution is a finite sum of spherical harmonics, we have:

$$
w_{p+1}=0
$$


Hence, in this case, the boundary condition defined by $p$ auxiliary functions is exact. Therefore it is an exact, local boundary condition in space and time in the same sense that the conditions proposed by Grote and Keller [3,4] are exact and temporally local. The necessity of a spherical harmonic expansion in the Grote-Keller formulation probably renders theirs less efficient than the formulation proposed here. We note that a distinct formulation with the possibility of avoiding the spherical harmonic expansion is mentioned in their work, but is not implemented.

\section{The Two-Dimensional Maxwell System}

We now consider the generalization of this technique to a hyperbolic system. Namely, we study the two-dimensional Maxwell system in the TM case, given by:

$$
\frac{\partial}{\partial t}\left(\begin{array}{c}
E_{z} \\
H_{x} \\
H_{y}
\end{array}\right)=R(\theta) \frac{\partial}{\partial r}\left(\begin{array}{c}
E_{z} \\
H_{x} \\
H_{y}
\end{array}\right)+\frac{1}{r} \Theta(\theta) \frac{\partial}{\partial \theta}\left(\begin{array}{c}
E_{z} \\
H_{x} \\
H_{y}
\end{array}\right)
$$

where

$$
R=\left(\begin{array}{ccc}
0 & -\frac{1}{\epsilon} \sin \theta & \frac{1}{\epsilon} \cos \theta \\
-\frac{1}{\mu} \sin \theta & 0 & 0 \\
\frac{1}{\mu} \cos \theta & 0 & 0
\end{array}\right), \Theta=\left(\begin{array}{ccc}
0 & -\frac{1}{\epsilon} \cos \theta & -\frac{1}{\epsilon} \sin \theta \\
-\frac{1}{\mu} \cos \theta & 0 & 0 \\
-\frac{1}{\mu} \sin \theta & 0 & 0
\end{array}\right) .
$$

Note that it is possible to directly apply results for the wave equation to this system as in [5]. However, it is instructive to proceed directly. Setting $c=(\epsilon \mu)^{-1 / 2}$, the progressive wave expansion now takes the form:

$$
\left(\begin{array}{c}
E_{z} \\
H_{x} \\
H_{y}
\end{array}\right)=\sum_{k=0}^{\infty} r^{-(k+1 / 2)} f_{k}(c t-r) a_{k}(\theta)
$$

where

$$
\begin{gathered}
f_{k}^{\prime}=f_{k-1}, \\
(c I+R) a_{0}=0, \\
(c I+R) a_{k}=-(k-1 / 2) R a_{k-1}+\Theta a_{k-1}^{\prime} .
\end{gathered}
$$

It is easily verified that the matrix on the left-hand side of (4.0.56) is singular, so that a nontrivial expansion exists. Generally one must solve an eikonal equation to determine the form of the expansion. A convenient orthonormal basis for expanding the solution is provided by:

$$
e_{1}=\left(\begin{array}{l}
1 \\
0 \\
0
\end{array}\right), \quad \rho=\left(\begin{array}{c}
0 \\
\cos \theta \\
\sin \theta
\end{array}\right), \quad \tau=\left(\begin{array}{c}
0 \\
-\sin \theta \\
\cos \theta
\end{array}\right)
$$


These satisfy the relations:

$$
\begin{gathered}
R e_{1}=\frac{1}{\mu} \tau, \quad R \rho=0, \quad R \tau=\frac{1}{\epsilon} e_{1} \\
\Theta e_{1}=-\frac{1}{\mu} \rho, \quad \Theta \rho=-\frac{1}{\epsilon} e_{1}, \quad \Theta \tau=0 \\
\rho^{\prime}=\tau, \quad \tau^{\prime}=-\rho .
\end{gathered}
$$

In terms of this basis, a right nullvector, $u$, and a left nullvector, $l$, of $(c I+R)$ are given by:

$$
u=e_{1}-\sqrt{\frac{\epsilon}{\mu}} \tau, l=e_{1}-\sqrt{\frac{\mu}{\epsilon}} \tau
$$

The solution of (4.0.56) is:

$$
a_{0}=A(\theta) u
$$

where $A(\theta)$ is an arbitrary function. For (4.0.57) we expand

$$
a_{k}=E_{k} e_{1}+\sqrt{\frac{\epsilon}{\mu}} B_{k} \rho+\sqrt{\frac{\epsilon}{\mu}} C_{k} \tau
$$

and note:

$$
E_{0}=-C_{0}=A, \quad B_{0}=0
$$

The equations become:

$$
\begin{gathered}
E_{k}+C_{k}=-\left((k-3 / 2) C_{k-1}+B_{k-1}^{\prime}\right) \\
E_{k}+C_{k}=-(k-1 / 2) E_{k-1} \\
B_{k}=-E_{k-1}^{\prime}
\end{gathered}
$$

Clearly, the system is solvable if and only if

$$
\left((k-3 / 2) C_{k-1}+B_{k-1}^{\prime}\right)=(k-1 / 2) E_{k-1},
$$

and then it only determines $E_{k}+C_{k}, B_{k}$. The complete determination of $E_{k}, C_{k}$ follows from the imposition of (4.0.69) at the next order. (Note that (4.0.69) does hold when $k=1$.) Solving these leads to the simple recursion relations for $k \geq 1$ :

$$
\begin{gathered}
E_{k}=-(2 k)^{-1}\left(E_{k-1}^{\prime \prime}+(k-1 / 2)^{2} E_{k-1}\right) \\
B_{k}=-E_{k-1}^{\prime}
\end{gathered}
$$




$$
C_{k}=(2 k)^{-1}\left(E_{k-1}^{\prime \prime}-\left(k^{2}-1 / 4\right) E_{k-1}\right) .
$$

To determine a boundary condition we first note that:

$$
\begin{gathered}
E_{z}=\sum_{k=0}^{\infty} r^{-(k+1 / 2)} f_{k}(c t-r) E_{k} \\
H_{r}=\cos \theta H_{x}+\sin \theta H_{y}=\sqrt{\frac{\epsilon}{\mu}} \sum_{k=0}^{\infty} r^{-(k+1 / 2)} f_{k}(c t-r) B_{k} \\
H_{\theta}=-\sin \theta H_{x}+\cos \theta H_{y}=\sqrt{\frac{\epsilon}{\mu}} \sum_{k=0}^{\infty} r^{-(k+1 / 2)} f_{k}(c t-r) C_{k}
\end{gathered}
$$

To leading order we use (4.0.65) to obtain:

$$
E_{z}+\sqrt{\frac{\mu}{\epsilon}} H_{\theta}=\sum_{k=1}^{\infty} r^{-(k+1 / 2)} f_{k}(c t-r)\left(E_{k}+C_{k}\right)=O\left(r^{-1}\right) \cdot\left|\left(E_{z}, H_{x}, H_{y}\right)\right| .
$$

A more accurate formula follows from taking the time derivative and using the recursions:

$\frac{1}{c} \frac{\partial}{\partial t}\left(E_{z}+\sqrt{\frac{\mu}{\epsilon}} H_{\theta}\right)=\sum_{k=0}^{\infty} r^{-(k+1 / 2)} f_{k}(c t-r)\left(E_{k+1}+C_{k+1}\right)=-\frac{1}{r} \sum_{k=0}^{\infty} r^{-(k+1 / 2)} f_{k}(c t-r)(k+1 / 2) E_{k}$,

which implies

$$
\frac{1}{c} \frac{\partial}{\partial t}\left(E_{z}+\sqrt{\frac{\mu}{\epsilon}} H_{\theta}\right)+\frac{1}{2 r} E_{z}=O\left(r^{-2}\right) \cdot\left|\left(E_{z}, H_{x}, H_{y}\right)\right| .
$$

To find higher order conditions, we must derive a relationship for the evolution of the right-hand side, $w_{1}$, of the equation above. For $j \geq 1$ set

$$
w_{j}=\sum_{k=j}^{\infty} r^{-k-j-1 / 2} f_{k} \gamma_{k, j} E_{k}=O\left(r^{-2 j}\right) \cdot\left|\left(E_{z}, H_{x}, H_{y}\right)\right| .
$$

Here $\gamma_{k, j}$ is again given by (2.0.13). Note that $w_{1}$ is indeed the right-hand side of (4.0.78). Differentiating $w_{j}$ with respect to $t$ and using (4.0.55),(4.0.70) yields:

$$
\begin{aligned}
\frac{1}{c} \frac{\partial w_{j}}{\partial t} & =\sum_{k=j}^{\infty} r^{-k-j-1 / 2} f_{k}^{\prime} \gamma_{k, j} E_{k} \\
& =\sum_{k=j-1}^{\infty} r^{-k-j-3 / 2} f_{k} \gamma_{k+1, j} E_{k+1} \\
& =-\frac{1}{2} \sum_{k=j-1}^{\infty} r^{-k-j-3 / 2} f_{k} \gamma_{k+1, j}(k+1)^{-1}\left(E_{k}^{\prime \prime}+(k+1 / 2)^{2} E_{k}\right)
\end{aligned}
$$


From the identity

$$
(k+1 / 2)^{2}=(k-(j-1))(k-j)+2 j(k-(j-1))+(j-1 / 2)^{2},
$$

and (2.0.13) we finally derive

$$
\frac{1}{c} \frac{\partial w_{j}}{\partial t}=\frac{1}{4 r^{2}}\left(\frac{\partial^{2} w_{j-1}}{\partial \theta^{2}}+(j-1 / 2)^{2} w_{j-1}\right)-\frac{j}{r} w_{j}+w_{j+1}
$$

Putting these together we have a boundary condition whose formal order of accuracy is $r^{-2 m}$ :

$$
\begin{gathered}
\frac{1}{c} \frac{\partial}{\partial t}\left(E_{z}+\sqrt{\frac{\mu}{\epsilon}} H_{\theta}\right)+\frac{1}{2 r} E_{z}=w_{1} \\
\frac{1}{c} \frac{\partial w_{j}}{\partial t}+\frac{j}{r} w_{j}=\frac{1}{4 r^{2}}\left(\frac{\partial^{2} w_{j-1}}{\partial \theta^{2}}+(j-1 / 2)^{2} w_{j-1}\right)+w_{j+1}, \quad j=1, \ldots, m-1, \\
w_{0}=2 E_{z}, \quad w_{m}=0 .
\end{gathered}
$$

Of course in numerical implementations, this condition must be supplemented with some extrapolation of the "outgoing" variable, $E_{z}-\sqrt{\frac{\mu}{\epsilon}} H_{\tan }$ and a computation of the variable characteristic along the boundary, $H_{\text {normal }}$. (For a circular boundary these are $H_{\text {tan }}=H_{\theta}$, $H_{\text {normal }}=H_{r}$.) A three-dimensional version also exists. Moreover, it is exact in the same way that the condition described in the previous section is exact. See [7] for more details.

\section{Numerical Experiments}

We consider numerical experiments with these conditions in the case of the two-dimensional wave equation with $c=1$. Further calculations along with experiments for Maxwell's equations are in progress [7]. The test case considered here is a Dirichlet problem exterior to a disk of unit radius. A range of tangential Fourier modes is excited by a time-dependent amplitude function which both oscillates and decays. Precisely we take:

$$
u(r=1, \theta, t)=\frac{1-\cos 2 \pi t}{1+t^{2}} \cos n \theta,
$$

for $n=0,5,10,25,50$. The modal wave equation governing the amplitude of $\cos n \theta$, that is:

$$
\frac{\partial^{2} u}{\partial t^{2}}=\frac{\partial^{2} u}{\partial r^{2}}+\frac{1}{r} \frac{\partial u}{\partial r}-\frac{n^{2}}{r^{2}} u
$$

is discretized by a standard second order central difference scheme in space-time and the boundary conditions given by equations $(2.0 .17)-(2.0 .18)$ are discretized as described below. 
To construct a second order approximation to these equations, we apply the boundary condition half way between the last two mesh circles and we also use the fact that $w_{j+1}$ is small compared to $w_{j},\left(w_{1}\right.$ small compared to $\left.u\right)$. Then we approximate $(2.0 .17)$ by:

$$
\begin{aligned}
\frac{1}{2 \delta t}\left(\left(u_{N}^{t^{+}}+u_{N-1}^{t^{+}}\right)-\left(u_{N}^{t}+u_{N-1}^{t}\right)\right) & +\frac{1}{2 \delta r}\left(\left(u_{N}^{t^{+}}+u_{N}^{t}\right)-\left(u_{N-1}^{t^{+}}+u_{N-1}^{t}\right)\right) \\
& +\frac{1}{8 R}\left(u_{N}^{t^{+}}+u_{N-1}^{t^{+}}+u_{N}^{t}+u_{N-1}^{t}\right) \\
& =\frac{3}{2} w_{1}^{t}-\frac{1}{2} w_{1}^{t^{-}}
\end{aligned}
$$

and (2.0.18) by

$$
\begin{aligned}
\frac{1}{\delta t}\left(w_{j}^{t^{+}}-w_{j}^{t}\right) & +\frac{j}{2 R}\left(w_{j}^{t^{+}}+w_{j}^{t}\right) \\
& =\frac{(j-1 / 2)^{2}-n^{2}}{8 R^{2}}\left(w_{j-1}^{t^{+}}+w_{j-1}^{t}\right) \\
& +\frac{3}{2} w_{j+1}^{t}-\frac{1}{2} w_{j+1}^{t^{-}} .
\end{aligned}
$$

For $w_{0}$ we use the sum of $u$ at $N$ and $N-1$. Note that although the system would formally be implicit in the $\theta$-derivative approximation for a non-modal algorithm, no matrix factorizations are involved if we solve sequentially. That is, $w_{j-1}^{t^{+}}$is computed before its $\theta$-difference is used to update $w_{j}$. The calculations below are for $t=15$ with varying artificial boundary location $R$. The value of $m$ is the number of auxiliary functions used, so that the formal asymptotic order is $2 m+2$. The value of $\delta r$ is chosen to yield about four digits of accuracy in the numerical solution. We are thus forced to use a much finer mesh for $n=50$ than for $n=0$, with the number of points increasing by a factor of 30 . The 'exact' solution is computed with the same mesh spacing but $R=18$. In all cases we choose $\delta t=0.8 \delta r$. Errors were measured at $t=.5,1,1.5, \ldots, 15$. The time station at which the maximum error occurred, not included in the tables, was always $t=15$ for $n=0$, but earlier in the other cases. The largest error was, however, always recorded after the waves with largest amplitude passed through the artificial boundary. We note that at later times the solution is fairly small, so that the relative $L_{2}$ error criterion used here is quite strict. Its precise definition is:

$$
E^{2}(t)=\frac{\sum_{j}\left(u_{\text {long }}\left(r_{j}, t\right)-u\left(r_{j}, t\right)\right)^{2}}{\sum_{j}\left(u_{\text {long }}\left(r_{j}, t\right)\right)^{2}}
$$

We first consider $n=0$ where $\delta r=5 \times 10^{-3}$. 
Maximum Relative Error $n=0$

\begin{tabular}{|c||c|c|c|}
\hline$m$ & $R=2$ & $R=3$ & $R=4$ \\
\hline 0 & $3.8 \times 10^{-1}$ & $5.0 \times 10^{-1}$ & $5.4 \times 10^{-1}$ \\
\hline 2 & $2.2 \times 10^{-1}$ & $1.2 \times 10^{-1}$ & $4.0 \times 10^{-2}$ \\
\hline 5 & $2.9 \times 10^{-2}$ & $3.1 \times 10^{-3}$ & $1.7 \times 10^{-4}$ \\
\hline 10 & $4.4 \times 10^{-4}$ & $1.2 \times 10^{-4}$ & $1.7 \times 10^{-4}$ \\
\hline 20 & $4.6 \times 10^{-5}$ & $1.2 \times 10^{-4}$ & $1.7 \times 10^{-4}$ \\
\hline
\end{tabular}

The second case is given by $n=5$ with $\delta r=2.5 \times 10^{-3}$.

Maximum Relative Error $n=5$

\begin{tabular}{|c||c|c|c|}
\hline$m$ & $R=2$ & $R=3$ & $R=4$ \\
\hline 0 & $5.0 \times 10^{-1}$ & $4.5 \times 10^{-1}$ & $4.0 \times 10^{-1}$ \\
\hline 2 & $2.3 \times 10^{-2}$ & $6.0 \times 10^{-3}$ & $1.8 \times 10^{-3}$ \\
\hline 5 & $3.9 \times 10^{-5}$ & $6.6 \times 10^{-5}$ & $1.3 \times 10^{-4}$ \\
\hline 10 & $3.9 \times 10^{-5}$ & $6.6 \times 10^{-5}$ & $1.3 \times 10^{-4}$ \\
\hline 20 & $3.9 \times 10^{-5}$ & $6.6 \times 10^{-5}$ & $1.3 \times 10^{-4}$ \\
\hline
\end{tabular}

The third case is given by $n=10$ with $\delta r=1 . \overline{6} \times 10^{-3}$.

Maximum Relative Error $n=10$
\begin{tabular}{|c||c|c|c|}
\hline$m$ & $R=2$ & $R=3$ & $R=4$ \\
\hline 0 & 1.0 & 1.1 & 1.1 \\
\hline 2 & $3.0 \times 10^{-2}$ & $4.9 \times 10^{-3}$ & $1.5 \times 10^{-3}$ \\
\hline 5 & $5.7 \times 10^{-4}$ & $9.7 \times 10^{-5}$ & $2.0 \times 10^{-4}$ \\
\hline 10 & $3.9 \times 10^{-5}$ & $9.6 \times 10^{-5}$ & $2.0 \times 10^{-4}$ \\
\hline 20 & $3.9 \times 10^{-5}$ & $9.6 \times 10^{-5}$ & $2.0 \times 10^{-4}$ \\
\hline
\end{tabular}

The fourth case is given by $n=25$ with $\delta r=3 . \overline{3} \times 10^{-4}$. 
Maximum Relative Error $n=25$

\begin{tabular}{|c||c|c|c|}
\hline$m$ & $R=2$ & $R=3$ & $R=4$ \\
\hline 0 & $5.4 \times 10^{-1}$ & $5.7 \times 10^{-1}$ & $5.7 \times 10^{-1}$ \\
\hline 2 & $4.5 \times 10^{-3}$ & $7.0 \times 10^{-4}$ & $1.9 \times 10^{-4}$ \\
\hline 5 & $2.1 \times 10^{-5}$ & $6.1 \times 10^{-5}$ & $1.2 \times 10^{-4}$ \\
\hline 10 & $2.1 \times 10^{-5}$ & $6.1 \times 10^{-5}$ & $1.2 \times 10^{-4}$ \\
\hline 20 & $2.1 \times 10^{-5}$ & $6.1 \times 10^{-5}$ & $1.2 \times 10^{-4}$ \\
\hline
\end{tabular}

Finally we take $n=50$ with $\delta r=1 . \overline{6} \times 10^{-4}$.

Maximum Relative Error $n=50$

\begin{tabular}{|c||c|c|c|}
\hline$m$ & $R=2$ & $R=3$ & $R=4$ \\
\hline 0 & $4.1 \times 10^{-1}$ & $4.5 \times 10^{-1}$ & $4.5 \times 10^{-1}$ \\
\hline 2 & $2.3 \times 10^{-3}$ & $3.7 \times 10^{-4}$ & $1.3 \times 10^{-4}$ \\
\hline 5 & $2.2 \times 10^{-5}$ & $6.6 \times 10^{-5}$ & $1.3 \times 10^{-4}$ \\
\hline 10 & $2.2 \times 10^{-5}$ & $6.6 \times 10^{-5}$ & $1.3 \times 10^{-4}$ \\
\hline 20 & $2.2 \times 10^{-5}$ & $6.6 \times 10^{-5}$ & $1.3 \times 10^{-4}$ \\
\hline
\end{tabular}

With increasing $m$ we rapidly drive the error down to the level of the discretization error, so that the results become $m$-independent. Note that this final error is not zero as we are comparing with a numerical long domain solution. Indeed, the final error (as $m \rightarrow \infty$ ) may increase with increasing domain size. We further checked the $m$-independence of the results by carrying out additional experiments with $m=30,40,50,60$, which produced errors identical to the $m=20$ case shown below. Note that for none of the domains tested were the $m=0$ results acceptable. For $m=20$, in contrast, the errors are dominated by discretization errors even on the smallest domain. Generally, the $n=0$ case is the most difficult, as predicted by the analysis of [9].

\section{Concluding Remarks}

We believe the boundary conditions formulated in this paper provide an easily implementable path to high accuracy, at a computational cost which is favorable in comparison with currently available alternatives. There are, however, some open issues to be addressed. From a theoretical perspective, asymptotic error estimates are as yet unproven in the two-dimensional case, 
and one as always expects some time nonuniformity in the approximation. From a practical perspective, our current formulation is restricted to circular/spherical boundaries. It would, of course, be useful to have more flexibility. The expansions themselves should be valid outside some circle/sphere containing sources, scatterers and other perturbations. However, the direct imposition of the conditions on a boundary of different shape requires normal derivatives of the auxiliary functions. It would then be necessary to define and store them off the boundary, which we want to avoid for reasons of cost and complexity. Generalizations to anisotropic problems such as the convective wave equation and the linearized compressible Euler equation are also possible. Now the boundary must take a special shape determined by the eikonal equation associated with the underlying velocity field. This will be discussed in detail in a later paper.

\section{References}

[1] A. Barry, J. Bielak and R.C. MacCamy, " On Absorbing Boundary Conditions for Wave Propagation", J. Comp. Phys., 79 (1988), 449-468.

[2] A. Bayliss and E. Turkel, "Radiation Boundary Conditions for Wave-Like Equations", Comm. Pure and Appl. Math., 33 (1980), 707-725.

[3] M. Grote and J. Keller, "Exact Nonreflecting Boundary Conditions for the Time Dependent Wave Equation", SIAM J. Appl. Math., 55 (1995), 280-297.

[4] M. Grote and J. Keller, "Nonreflecting Boundary Conditions for Time Dependent Scattering", J. Comp. Phys., 127 (1996), 52-81.

[5] T. Hagstrom, B. Alpert, L. Greengard and S.I. Hariharan, "Accurate Boundary Treatments for Maxwell's Equations and their Computational Complexity", 13th Annual Review in Applied Computational Electromagnetics, to appear.

[6] T. Hagstrom and S.I. Hariharan, "Progressive Wave Expansions and Asymptotic Boundary Conditions for Hyperbolic Systems", in preparation.

[7] T.Hagstrom, S.I. Hariharan, and D. Watkins, "Numerical Solutions of Maxwell's Equations in Unbounded Regions" (in preparation).

[8] T. Hagstrom and S.I. Hariharan, "Progressive Wave Expansions and Open Boundary Problems", IMA Volumes in Mathematics and its Applications - Computational Wave Propagation, Vol. 86, (1997), Eds. B. Engquist and G. A. Kriegsmann, Springer Verlag, pp. 23-43.

[9] T. Hagstrom, S.I. Hariharan and R.C. MacCamy, "On the Accurate Long-Time Solution of the Wave Equation in Exterior Domains: Asymptotic Expansions and Corrected Boundary Conditions", Math. Comp., 63 (1994), 507-539. 



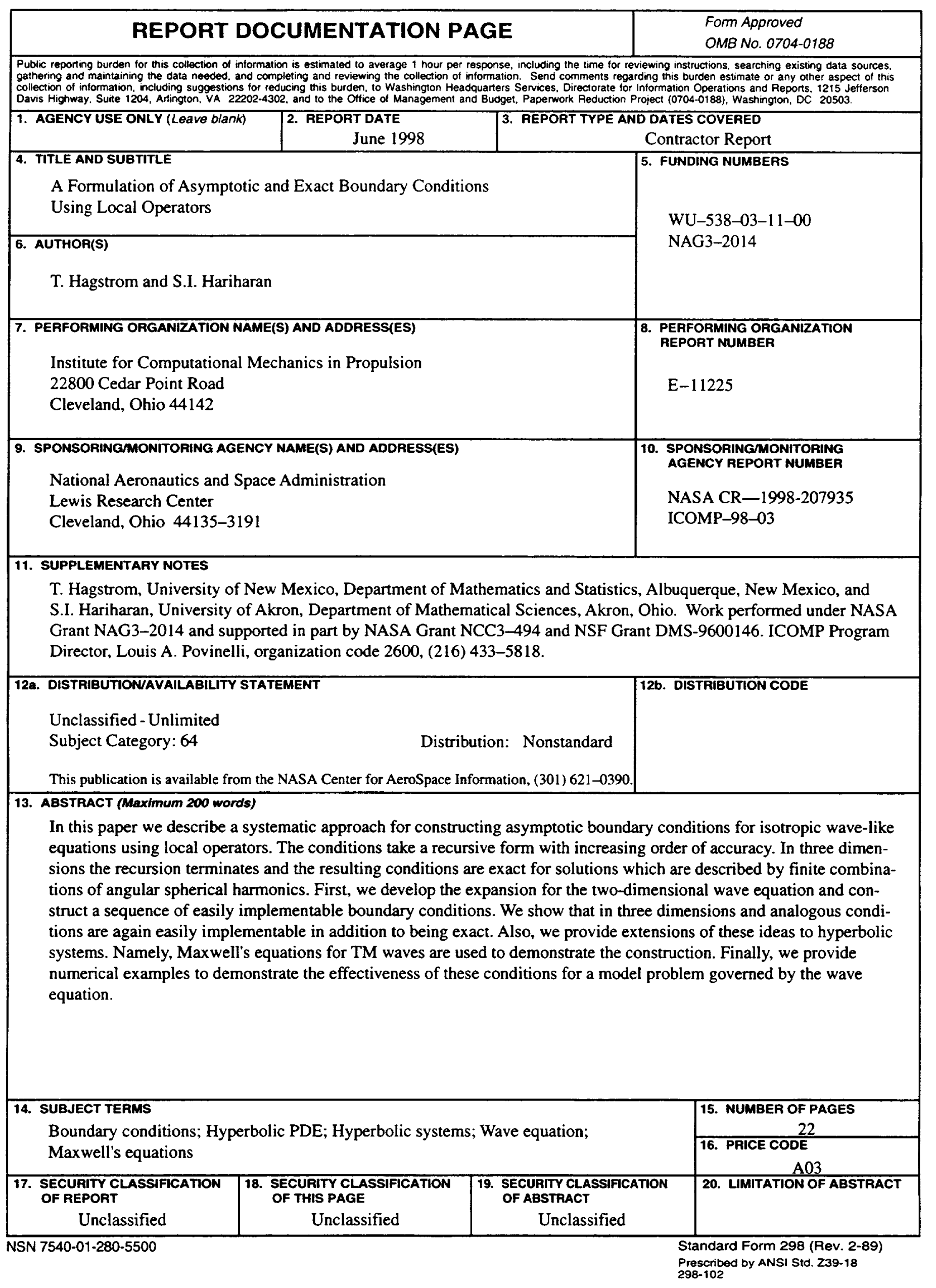

